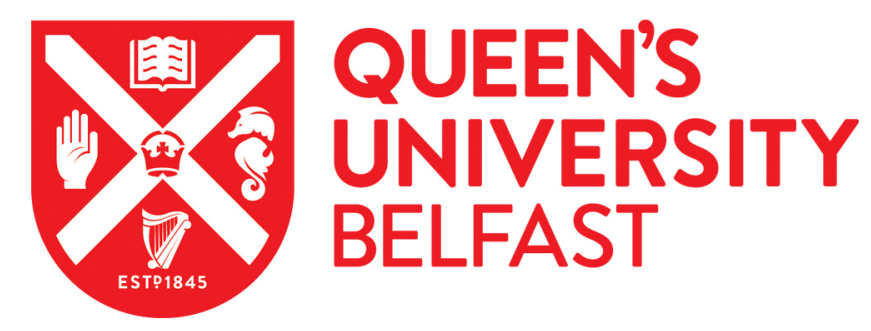

\title{
Manufacturable Ultra-Thin Resistive FSS for Challenging EM Mobile Environments
}

Zabri, S., Cahill, R., Conway, G., McGuigan, N., Zelenchuk, D., \& Dickie, R. (2018). Manufacturable Ultra-Thin Resistive FSS for Challenging EM Mobile Environments. In Proceedings of the 12th European Conference on Antennas and Propagation (EuCAP) 9-12 April 2018

Published in:

Proceedings of the 12th European Conference on Antennas and Propagation (EuCAP) 9-12 April 2018

Document Version:

Peer reviewed version

Queen's University Belfast - Research Portal:

Link to publication record in Queen's University Belfast Research Portal

Publisher rights

Copyright 2018 IEEE This work is made available online in accordance with the publisher's policies. Please refer to any applicable terms of use of the publisher.

\section{General rights}

Copyright for the publications made accessible via the Queen's University Belfast Research Portal is retained by the author(s) and / or other copyright owners and it is a condition of accessing these publications that users recognise and abide by the legal requirements associated with these rights.

Take down policy

The Research Portal is Queen's institutional repository that provides access to Queen's research output. Every effort has been made to ensure that content in the Research Portal does not infringe any person's rights, or applicable UK laws. If you discover content in the Research Portal that you believe breaches copyright or violates any law, please contact openaccess@qub.ac.uk. 


\title{
Manufacturable Ultra-Thin Resistive FSS for Challenging EM Mobile Environments
}

\author{
S.N Zabri ${ }^{1}$, R. Cahill², G. Conway ${ }^{2}$, N. McGuigan ${ }^{2}$, D. Zelenchuk ${ }^{2}$, R. Dickie ${ }^{2}$ \\ ${ }^{1}$ Universiti Teknikal Malaysia Melaka, 76100 Durian Tunggal, Melaka, Malaysia, email: normi@utem.edu.my \\ ${ }^{2}$ Queen's University of Belfast, ECIT, Belfast, Northern Ireland, UK, email: r.cahill@qub.ac.uk
}

\begin{abstract}
This paper reports on recent developments in the manufacture and measured electromagnetic (EM) performance of a new class of thin microwave absorbers based on metal backed resistively loaded Frequency Selective Surfaces (FSS). Ink jet printing technology is shown to be a viable method for creating patterned FSS elements very close to the desired resistance values. This is demonstrated by comparing simulated and measured reflectivity plots for an ink jet and a stencil printed polarisation independent absorber that is designed to work in the frequency range $7.1-24 \mathrm{GHz}$. Numerical simulations show that thin FSS absorbers provide radar backscatter suppression, not only in the far field of the illuminating antenna, but also when the EM source is placed very close to the surface of the periodic array. This technology can therefore provide a solution for enhancing the performance of wireless sensors placed close to lossy loads such as the human body.
\end{abstract} sensors

Index Terms-FSS, microwave absorbers, radar, on body

\section{INTRODUCTION}

Radar cloaking of structures such as aircraft, vehicle surfaces and wind turbines require the deployment of thin and light weight microwave absorbent material such as a metal backed resistively loaded FSS [1-2]. For these structures, the shape and size of the individual elements of the periodic array and the separation distance to the metal ground plane are critical design factors that determine the frequency of operation, bandwidth and sensitivity to the angle and polarisation of the impinging wave. Optimisation of the geometry can be made using a numerical simulator such as CST Microwave Studio, such that at resonance, the inductance presented by the ground plane of the structure (thickness $<\lambda / 4$ ), is used to cancel the imaginary component of the FSS reactance. When FSS are designed to provide spatial filtering, the conductivity of the metal patterned elements is required to be very large to avoid transmission and/or reflection losses, particularly around the desired resonant frequencies [3]. On the contrary the individual elements in the unit cells of metal backed FSS absorbers should be designed to present small surface resistance values generally in the range $13-175 \Omega / \mathrm{sq}$ [4]. The exact value is selected to impedance match the structure to free space $(377 \Omega)$. Physically small technology demonstrators with lumped resistors loading loop elements have been used in proof of concept studies as a means to validate numerical results [5], however this approach is undesirable for 'real world' applications, principally because of the component and manufacturing costs. An alternative strategy that offers a potentially better solution for low cost mass production of microwave absorbers is discussed in this paper. We show that values close to the desired load resistances can be achieved by the stencil deposition of graphite and carbon based paints, but more repeatable and precise results are obtained using ink jet printing using doped nano silver ink mixtures. Both fabrication techniques are discussed in this paper and measured reflectivity plots obtained for a stencil and an ink-jet manufactured absorber that was designed to operate in the $7.1-24 \mathrm{GHz}$ frequency range are compared with numerical simulations.

Previously reported numerical and experimental results for this class of absorber are based on the assumption that the absorber is positioned in the far field of the single (monostatic) or two (bistatic) antennas that are used to illuminate and measure the backscattered signals. However, in this paper we show that the structure also works as a reasonably efficient absorber when the antenna is placed very close to the surface of the FSS. This is demonstrated in the $10-11 \mathrm{GHz}$ frequency range by observing the detuning of an impedance matched microstrip patch antenna, which occurs when the radiating aperture is located at distances of between $1 \mathrm{~mm}$ and $5 \mathrm{~mm}$ above the resistively loaded FSS absorber. The small change in impedance which is observed, suggests that this technology might offer a solution for challenging EM mobile environments such as improving the performance of on-body sensors.

\section{DESIGN OF THE FSS ABSORBER}

The absorber design previously reported by the authors in [6] was employed for the experimental (section III and IV) and numerical studies (section V) which are presented in this paper. A schematic of the unit cell geometry and physical dimensions are given in Fig. 1. The periodic array which is patterned on a $0.13 \mathrm{~mm}$ thick substrate $\left(\varepsilon_{\mathrm{r}}=2.2\right.$, $\tan \delta=0.0009$ ) and positioned $3.1 \mathrm{~mm}$ above a metal ground plane with an air gap separating the two layers, was optimised to provide a $-10 \mathrm{~dB}$ reflectivity bandwidth of $108 \%(7.1-24 \mathrm{GHz})$ at normal incidence. The unequal width of the hexagonal loops was selected to permit a single surface resistance value $(27 \Omega /$ sq.) to be used for the design 


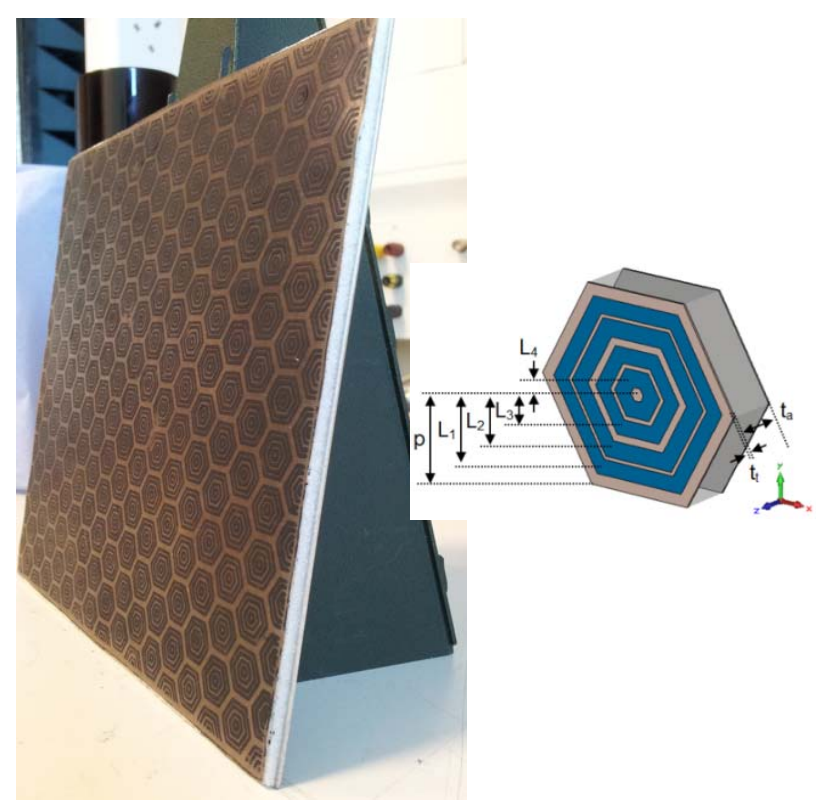

Fig. 1. Photograph of the manufactured metal backed FSS absorber and schematic and dimensions of the FSS unit cell; $\mathrm{p}=6.9 \mathrm{~mm}, \mathrm{~L}_{1}=5.6 \mathrm{~mm}$, $\mathrm{L}_{2}=4.2 \mathrm{~mm}, \mathrm{~L}_{3}=2.5 \mathrm{~mm}, \mathrm{~L}_{4}=1.1 \mathrm{~mm}, \mathrm{w}_{1}=1.1 \mathrm{~mm}, \mathrm{w}_{2}=1.2 \mathrm{~mm}, \mathrm{w}_{3}=1.1 \mathrm{~mm}$, $\mathrm{W}_{4}=1.2 \mathrm{~mm}, \mathrm{R}=27 \Omega / \mathrm{sq}, \mathrm{t}_{\mathrm{a}}=3 \mathrm{~mm}, \mathrm{t}_{\mathrm{t}}=0.13 \mathrm{~mm}$

of the structure, thereby simplifying the manufacture of the periodic array. Fig. 2 depicts the reflectivity plot computed using CST Microwave Studio software, at normal incidence, where the symmetry afforded by the tightly coupled geometry of the periodic array, is polarisation independent.

\section{ABSORBER MANUFACTURE}

The method normally employed for creating resistively loaded FSS elements is to strategically insert lumped resistors, normally at high current positions, across narrow gaps inserted in the surface of loop elements [5]. For practical applications, the use of lump resistors is feasible at lower microwave frequencies but the cost and manufacturing complexity is prohibitive when this method is employed to create a large array of loops for wide bandwidth operation, such as the one shown in Fig. 1. Moreover for more advanced architectures, such as multilayer FSS, where the sheets containing the elements are bonded closely together, the presence of lumped resistors would complicate the manufacturing process and likely introduce undesirable voids between the individual surfaces. To overcome these manufacturing limitations, a viable solution is to pattern the gaps between the elements [2], or for additional polarisation functionality the whole surface of the features, with a material that exhibits a conductivity that is slightly lower than bulk metals. The precise surface resistance value equivalent to lumped resistive loading can be obtained from numerical

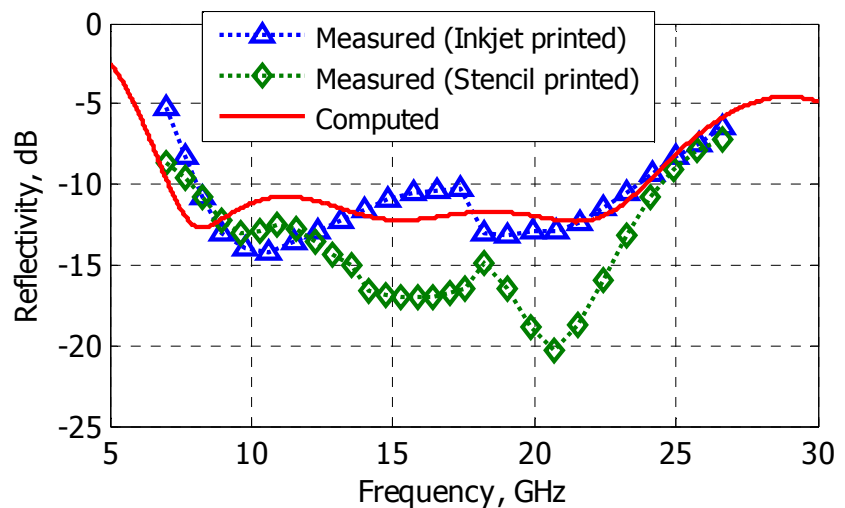

Fig. 2. Simulated and measured reflectivity plots at normal incidence

simulations, which for the absorber illustrated in Fig. 1, is $27 \Omega /$ sq. Two experimental printing techniques are described in the following subsections and the measured performance of each $15 \times 15 \mathrm{~cm}^{2}$ structure is compared with the predicted reflectivity plot in Fig. 2 to establish the preferred solution.

\section{A. Stencil Printed FSS Absorber}

The double periodic array with $13 \times 16$ unit cells is patterned on a $0.13 \mathrm{~mm}$ thick Taconic TLY-5 microwave laminate $\left(\varepsilon_{\mathrm{r}}=2,2, \tan \delta=0.0009\right)$ and bonded to a $3 \mathrm{~mm}$ thick Rohacell aluminum backed spacer. Y Shield HSF-74 electro conductive shielding paint was used to form the elements on the surface of the laminate by transferring the array pattern onto a $4 \mathrm{~mm}$ thick Linoleum sheet as depicted in Fig. 3. HSF-74 is a graphite and carbon based material, which is reinforced with long conductive fibers, providing up to $36 \mathrm{~dB}$ signal attenuation at frequencies up to $18 \mathrm{GHz}$ when applied at a coverage rate of $7.5 \mathrm{~m}^{2} / 1$ [7]. In the first step, the template is made by patterning the array on the surface of the Linoleum sheet using a milling machine. The gaps are made as deep as possible $(1 \mathrm{~mm})$ to prevent the spilling of ink, which would cause electrical connections between the individual loops in the unit cells. The HSF-74 is rolled on to the surface of the template using an ink roller and finally the hexagonal loop pattern is transferred on to the $0.13 \mathrm{~mm}$ thick substrate. The ink thickness was selected to be $43 \mu \mathrm{m}$ in order to obtain the required $27 \Omega /$ sq based on the thickness and surface resistance relationship plot reported in [8]. During manufacture it proved very difficult to tightly control the ink deposition and to realign the template with the first patterned layer to increase the thickness, therefore trial and error was used to obtain a value close to the desired result. Physical measurements of the average paint film thickness were made using a Tencor Alpha-Step 200 Profilometer. 
1. $15 \times 15 \mathrm{~cm}^{2}$ FSS pattern ( $1 \mathrm{~mm}$ deep) is etched onto a linoleum sheet (4 mm) and HSF-74 ink is rolled onto the template surface
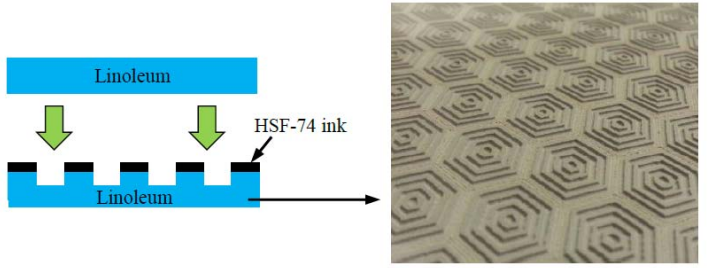

2. A patterned template with HSF-74 ink is transferred onto a $0.13 \mathrm{~mm}$ thick Taconic TLY-5 substrate

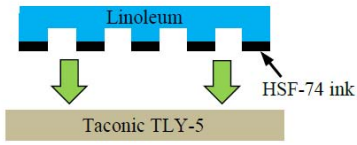

3. The final structure of the fabricated FSS absorber consists of hexaqonal loops
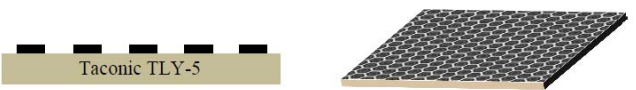

Fig. 3. Stencil printing manufacturing steps

\section{B. Ink Jet Printed FSS Absorber}

In [4] the authors proposed an alternative means to create resistively loaded elements of a FSS, which overcomes the limitations identified during the prototyping of the stencil patterned structures, namely the minor variations of the micron thick HSF-74 paint layers with measured values that were found to be between $20 \%$ [2] and 30\% [6] higher than the nominal design value for optimum computed backscatter response. An ink jet printer can be employed to simultaneously pattern the FSS elements on the substrate and digitally control the dot density of an aqueous and nano silver ink mixture to obtain surface resistance values that are much closer to the specified values for optimum absorber performance. Using spectral transmission and reflection measurements, plots showing the relationship between the surface resistance and the dot density in conjunction with the fraction of silver nano particles in the ink composition, enables engineering of the desired load values [4]. This simple, rapid and low cost method for printing FSS elements with material that has a slightly lower conductivity than metals was used to fabricate the structure shown in Fig. 1. The periodic array was printed on a $0.14 \mathrm{~mm}$ thick Novelle IJ-220 substrate using a 1:7 mixture composition $\left(\right.$ ink $_{\mathrm{ml}}$ :Metalon ${ }^{\mathrm{TM}}$ aqueous vehicle $\mathrm{ml}_{\mathrm{ml}}$ ). The ink solution was prepared and used in an Epson Stylus C88+ inkjet printer configured to provide a single pass trace and operate with a single ink cartridge and digitally configured for black and white printing and best photo quality resolution with a dot density corresponding to a RGB printer setting $(21,21,21)$.

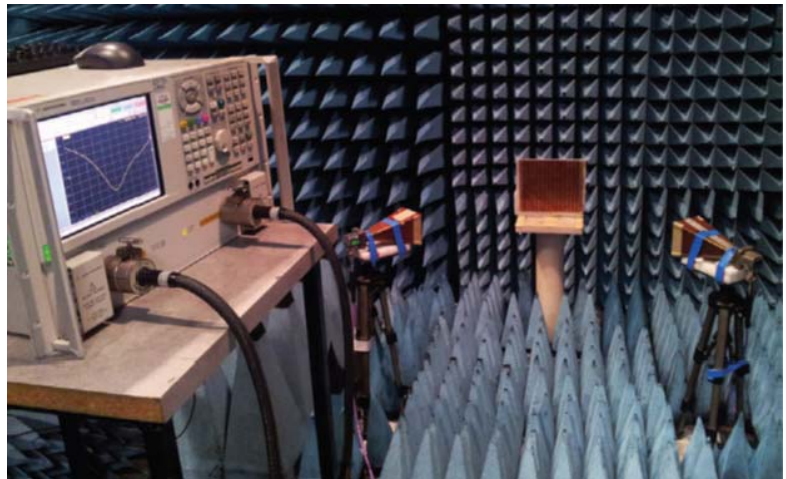

Fig. 4. Radar backscatter measurement set up ( $45^{\circ}$ incidence arrangement)

This dot density/ink mixture ratio gives a surface resistance value of $27 \Omega /$ sq. which was found in the simulations to maximize the reflectivity bandwidth of the FSS absorber. As in the stencil printed structure the periodic array was glued on the surface of a $3 \mathrm{~mm}$ thick Rhoacell metal backed spacer.

\section{MEASURED RESUlts AND OBSERVATIONS}

Time gated bi-static reflection measurements were carried out in an anechoic chamber for normal incidence in the frequency range $7-27 \mathrm{GHz}$. Three pairs of standard gain pyramidal horns were used and positioned at a distance of $47 \mathrm{~cm}$ from the two $15 \times 15 \mathrm{~cm}^{2}$ samples. A metal plate of the same size was first used to calibrate the experimental set-up, and then substituted with the stencil and ink-jet printed resistively loaded FSS absorbers. A photograph of the set-up is depicted in Fig. 4. Good agreement is shown in Fig. 2 between the CST computations and the measured reflectivity plots for both manufactured absorbers. The stencil printed structure exhibits a slightly smaller $-10 \mathrm{~dB}$ reflectivity bandwidth of $102 \%$ at normal incidence, and in general the experimental reflectivity levels are lower than those depicted in the simulated plots. Closer agreement is obtained when the surface resistance in the computer model is increased to $35 \Omega /$ sq (not shown for brevity). As a general observation, the performance of the resistively loaded hexagonal FSS absorber was optimised for TE and TM polarisations, and operation at incident angles ranging between $0^{\circ}$ and $45^{\circ}$. The requirement for the FSS to present a surface resistance of $27 \Omega / \mathrm{sq}$ is a compromise solution i.e. a slightly inferior performance at normal incidence is accepted in order to obtain a satisfactory radar backscatter response at oblique incidence. The measured reflectivity plot for the metal backed inkjet printed FSS is in much better agreement with the simulated data in terms of the radar backscatter levels within the operating frequency band, 7- $27 \mathrm{GHz}$. The results therefore confirm that this approach provides a better solution than stencil printing, for tightly controlling the surface resistance value of the manufactured FSS elements. 


\section{NEAR FIELD REQUIREMENT AND PERFORMANCE}

In common with other research findings reported in the open literature, the radar backscatter from the thin resistively loaded FSS absorber was simulated by applying appropriate boundary conditions, so that a single unit cell was used to model a periodic structure that is infinite in extent and illuminated by a plane wave. Similarly, experimental verification of the electromagnetic performance was obtained by illuminating the FSS in the far field of the two feedhorns, as illustrated in Fig. 4. Maintaining high radar backscatter suppression when the illuminating antenna is placed in close proximity to the periodic array has major implications, which would yield a step change in the RF performance and flexibility of antennas for future wireless applications needed to support healthcare. Wearable medical applications have distinctive requirements in that the devices have to be ultrathin and unobtrusive to the wearer while preserving good electrical characteristics, foremost among these is a good impedance match stability [9]. Reliable, repeatable performance remains a challenge for close fitting printed wearable antennas, which experience significant variation, due to a wide range in tissue morphology and electrical characteristics, namely; permittivity and conductivity, between individuals [10]. An obvious solution is to deploy an electromagnetic barrier to decouple the antenna from the human body. In order to explore the possibility of deploying these structures to create a truly conformal solution at commercially exploitable manufacturing costs, we have studied the impact on the impedance match of an antenna that is placed very close to the surface of the $3 \mathrm{~mm}$ thick resistively loaded FSS absorber and compared the results with those obtained for a scattering surface composed of a simple metal sheet with the same physical area. As a general observation the lower operating band of the absorber is much higher in frequency than the ISM band, but the upward scaling of the physical dimensions does not apply to the absorber thickness which is optimised for a bandwidth of $108 \%$, about 30 times larger than that specified for medical sensors in ISM bands. Given that the bandwidth of the absorber is proportional to the electrical thickness, the latter can therefore be significantly reduced. The schematic in Fig. 5 shows the build model of the microstrip patch which will be used for the measurements, and the periodic array of the hexagonal FSS. The antenna employs two quarter wave transformers to impedance match the patch radiator to the 50 Ohm input port and is designed to give a return loss of $23 \mathrm{~dB}$ at $10.22 \mathrm{GHz}$. The copper layout of the transmission lines and antenna are printed on a $254 \mu \mathrm{m}$ thick substrate with relative permittivity of about 2.2 and loss tangent 0.0009 (RT/Duroid 5880). Fig. 6 shows numerical simulations of the return loss with the aperture of the antenna positioned at distances of 1, 2, 3, 4, and $5 \mathrm{~mm}$ above the surface of a 62 $\mathrm{mm} \times 48 \mathrm{~mm}$ metal sheet and a similar size $3 \mathrm{~mm}$ thick FSS absorber with 18 complete unit cell. The size reduction of the scattering surfaces in the build model of CST is made to reduce computing time and mirror the actual geometry of the experimental set-up with the metal sheet and FSS absorber

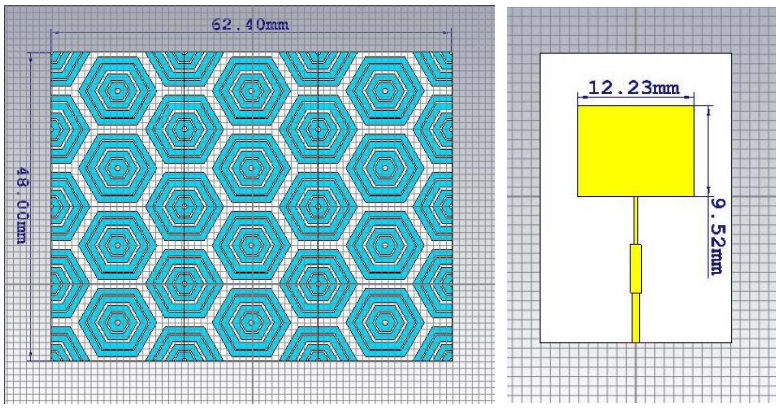

Fig. 5. Numerical build model of the microstrip antenna and the surface of the hexagonal FSS absorber.

(a)

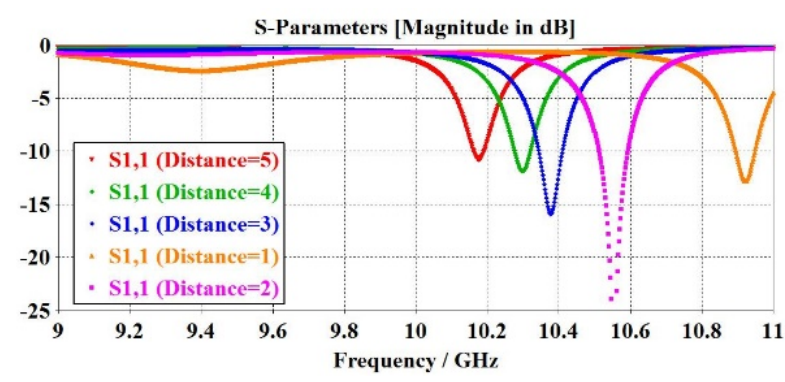

(b)

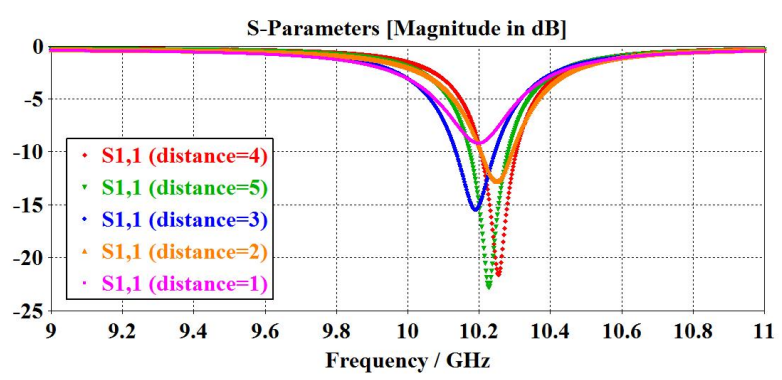

Fig. 6. Computed return loss of microstrip antenna placed at distance of $1,2,3,4$ and $5 \mathrm{~mm}$ above (a) the surface of the metal plate, (b) the surface of a resistively loaded hexagonal loop FSS absorber.

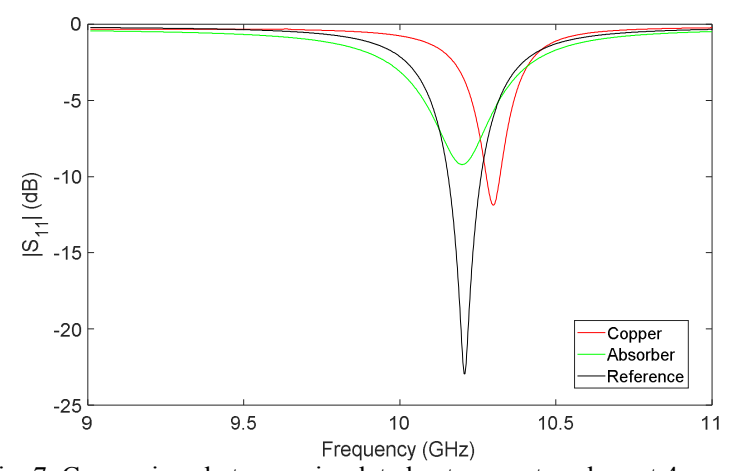

Fig. 7. Comparison between simulated antenna return loss at $4 \mathrm{~mm}$ distance from the metal ground plane with and without the resistively loaded FSS inserted $1 \mathrm{~mm}$ from the aperture of the microstrip patch. Antenna in isolation is the reference plot. 
placed on the surface of a precision 3D planar scanner. As expected significant detuning occurs when the separation distance between the antenna and metal sheet isvery small. For example for a $1 \mathrm{~mm}$ gap an upward shift of about 700 $\mathrm{MHz}(7 \%)$ is observed, but the effect is less significant for larger separations and the resonant frequency approaches the S11 response for the antenna in isolation, which should occur at about $7 \mathrm{~mm}(\lambda / 4)$. Much less detunning is predicted when the antenna is placed above the FSS absorber, but although the frequency shift is very small, the reflection coefficient minimum is lower for separation distances of 1 $\mathrm{mm}$ and $2 \mathrm{~mm}$, presumably because of the larger difference between the wave impedance at the surface of the absorber and the $377 \Omega$ that is presented by the structure. Fig. 7 shows a comparison between the $\left|S_{11}\right|$ prediction for the antenna placed $4 \mathrm{~mm}$ above the metal ground plane and $1 \mathrm{~mm}$ above the $3 \mathrm{~mm}$ thick absorber. For the same structure volume, the improvement in the stability of the resonant frequency of the antenna is apparent and the result demonstrates enhanced performance even at this relatively large antenna separation distance $(\lambda / 8)$. In this case when the metal plate is replaced by the FSS absorber, a $7 \mathrm{~dB}$ increase in the return loss of the antenna is predicted at $10.22 \mathrm{GHz}$.

\section{CONCLUSIONS}

The experimental results presented in this paper show that both stencil and ink jet printing techniques can be employed to create a thin resistively loaded metal backed FSS absorber. This is a simpler and lower cost manufacturing strategy compared to other previously published solutions based on the insertion of lumped resistors soldered across gaps in the loop elements. The main disadvantage of stencil patterning is attributed to the lack of control of the ink thickness, whereas inkjet printing provides a more repeatable digitally controlled means which was shown to provide surface resistances much closer to the desired values. Encouraging results were obtained from a preliminary feasibility study based on numerical simulations, which demonstrates the potential to deploy this class of absorber for enhanced performance of wireless sensors placed in close proximity to lossy loads such as the body. Integration of the antenna within the FSS absorber structure appears to be feasible and for this arrangement the metal ground plane would provide the necessary electromagnetic shielding. In future work to increase the Technology Readiness Level (TRL) of this concept, several major issues need to be addressed (i) a reduction in the thickness of the structure, which should be feasible given the less stringent bandwidth requirements and the possibility of using flexible material with a higher permittivity for the spacer, (ii) reduction in the area of the FSS required to give enhanced performance and (iii) modification of the FSS element shape and size and surface resistance to provide a better match (not $377 \Omega$ ) to the impedance of the incident wave in the near field of the antenna.

\section{REFERENCES}

[1] F. Costa, A. Monorchio, and G. Manara, " Analysis and design of ultra thin electromagnetic absorbers comprising resistively loaded high impedance surfaces," IEEE Trans. Antennas Propag., 2010, vol. 58, no. 5, pp. 1551-1558, 2010.

[2] S. N. Zabri, R. Cahill, and A. Schuchinsky, "Polarisation independent resistively loaded frequency selective surface absorber with optimum oblique incidence performance," IET Microwaves, Antennas Propag., vol. 8, no.14, pp. 1198-1203, 2014.

[3] R. Dickie, R. Cahill, H. Gamble, V. Fusco, and N. Mitchell, "THz frequency selective surface filters for Earth observation remote sensing instruments," IEEE Trans. Terahertz Science and Technology, vol. 1, no. 2, pp. 450-461, 2011.

[4] S. N. Zabri, R. Cahill, G. Conway and A. Schuchinsky, "Inkjet printing of resistively loaded FSS for microwave absorbers," Electron. Lett., vol. 51, no. 2, pp. 162-164, 2015.

[5] Y. Shang, Z. Shen, and S. Xiao, "On the design of single-layer circuit analog absorber using double-square-loop array," IEEE Trans. Antennas Propag., vol. 61, no.12, pp. 6022-6029, 2013

[6] S. N. Zabri, R. Cahill, and A. Schuchinsky, "Compact FSS absorber design using resistively loaded quadruple hexagonal loops for bandwidth enhancement," Electron. Lett., vol. 51, no. 2, pp. 162-164, 2015

[7] Y-SHIELD EMR Protection', http://www.yshield.com/, accessed January 2013

[8] S. N. Zabri, "Study of thin resistively loaded FSS based micriwave absorbers," PhD thesis, Queen's University Belfast, 2015

[9] P.S. Hall and Y. Hao, 'Antennas and Propagation for BodyCentric Wireless Communications,' Artech House Publisher, 2012, ISBN-13: 1-60807-376-4.

[10] G. A. Conway , W. G. Scanlon , C. Orlenius and C. Walker, "In-situ measurement of UHF wearable antenna efficiency using a reverberation chamber", IEEE Antennas Wireless Propag. Lett., vol. 7, pp.271-274, 2008 